\title{
Recursos didácticos virtuales en proyectos de ciencias naturales en período de confinamiento por COVID-19
}

\section{Virtual teaching resources in natural science projects in confinement period by COVID-19}

\author{
Maira Dolores Vélez-Loor \\ mvelez2802@pucem.edu.ec \\ Pontificia Universidad Católica del Ecuador, Extensión Manabí, Portoviejo \\ Ecuador \\ https://orcid.org/0000-0001-7742-8978 \\ Patricio Alfredo Vallejo-Valdivieso \\ pvallejo@pucem.edu.ec \\ patricio 2871@yahoo.es \\ Pontificia Universidad Católica del Ecuador, Extensión Manabí, Portoviejo \\ Ecuador \\ https://orcid.org/0000-0003-3248-7864 \\ María Elena Moya-Martínez \\ mmoya@pucem.edu.ec \\ Pontificia Universidad Católica del Ecuador, Extensión Manabí, Portoviejo \\ Ecuador \\ https://orcid.org/0000-0001-7504-6933
}

Recepción: 15 abril 2020

Revisado: 25 de mayo 2020

Aprobación: 13 junio 2020

Publicación: 16 de junio 2020

\section{RESUMEN}

La investigación tiene por objetivo analizar los recursos didácticos virtuales planificados para el abordaje de proyectos educativos de ciencias naturales en período de confinamiento por COVID-19. Generándose un trabajo de tipo descriptivo no experimental, con una población conformada por 30 docentes de la Unidad Educativa San Francisco Sales, ubicado en la Provincia de Manabí-Ecuador, siendo la muestra constituida por 6 docentes quienes cumplieron con los criterios de inclusión. Los resultados se abordaron desde las áreas: Recursos didácticos audiovisuales en redes sociales, Aulas virtuales, Método de aprendizaje, Plataformas de ciencias naturales. Los recursos didácticos virtuales proyectados por los docentes para ser implementados en el proyecto educativo de ciencias naturales, son idoneos 
para promover un aprendizaje significativo en los estudantes, sin embargo, es necesario contar con un proceso de capacitación tanto para profesores como discentes con la finalidad de evitar inconvenientes que perjudiquen el aprendizaje, así como el rendimiento escolar.

Descriptores: Aprendizaje activo; educación a distancia; tecnología educacional; epidemiología. (Palabras tomadas del Tesauro UNESCO).

\begin{abstract}
The objective of the research is to analyze the virtual didactic resources planned for the approach of educational projects in natural sciences during period of confinement by COVID-19. A non-experimental descriptive type work was generated, with a population made up of 30 teachers and a sample of 6 teachers who met the inclusion criteria at San Francisco Sales Educational Unit, located in the Province of ManabíEcuador. The results were approached from these areas: audiovisual teaching resources in social networks, virtual classrooms, learning method, and natural science platforms. The virtual didactic resources projected by the teachers to be implemented in the educational project of natural sciences, are ideal to promote significant learning in students, however, it is necessary to have a training process for both teachers and students in order to avoid disadvantages that impair learning, as well as school performance.
\end{abstract}

Descriptors: Activity learning; distance education; educational technology; epidemiology. (Words taken from the UNESCO Thesaurus).

\title{
INTRODUCCIÓN
}

Con fundamento en las corrientes pedagógicas, la didáctica en su esencia se ha encargado de facilitar el aprendizaje en los estudiantes. Es decir, busca crear una conexión directa entre la teoría y la práctica educativa por medio de la aplicación de adecuadas estrategias con el fin de promover una educación cónsona con la realidad social del estudiantes (Abreu, Gallegos, Jácome \& Martínez, 2017), lo cual permite diseñar diversos modelos de planificación educativa en correspondencia al enfoque epistémico abordado (Vallejo Valdivieso, Zambrano Pincay, Vallejo Pilligua \& Bravo Cedeño, 2019).

Siendo necesario considerar que el mundo se ha constituido en una aldea global donde la tendencia didáctica se perfila hacia la implementación de recursos virtuales 
(Pando, 2018), en América Latina, la ejecución de la educación virtual, así como el uso de los recursos didácticos asociados (Rama, 2016), se ha incrementado considerablemente, aumentando drásticamente por la emergencia de la pandemia de COVID-19 que ha obligado por prevención a tomar la educación virtual como alternativa para subsanar la planificación académica de la modalidad presencial, con la finalidad de concluir o iniciar el período según sea el caso, siendo posible además realizar por medio de las TIC, proyectos productivos y académicos (Lattá-Arias, 2019). En razón de la pandemia por COVID- 19, el Estado del Ecuador ha promulgado el Acuerdo Ministerial Nro. MDT-2020-076, mediante el cual se promueve el teletrabajo como opción obligatoria durante el tiempo que dure la emergencia sanitaria (Ministerio del Trabajo, 2020). De ese modo, el Ministerio de Educación (2020a) asumió la modalidad no presencial de estudios, gestionando la implementación obligatoria de recursos didácticos basados en lo virtual.

Destacándose que la virtualidad es una tendencia educativa a nivel global, la cual ha sido implementada en países llamados del primer mundo de modo efectiva, mas no ha sido una experiencia similar en los países latinoamericanos en donde se vienen gestionando su implementación progresivamente (Torres \& Rodríguez, 2019). Esto ha propiciado situaciones problemáticas por cuanto los docentes que tradicionalmente han laborado en modalidad presencial, se enfrentan al reto de asumir lo virtual obligatoriamente, viéndose contrariados en gestionar el aprendizaje desde esta vertiente educativa, y siendo necesario la conformación de equipos de trabajo que apoyen al docente en la consecución de tal fin, resultando ser los representantes un factor aliado (Hernández, 2018).

De allí que sea pertinente conocer las impresiones de los actores educativos sobre el uso de recursos didácticos con la finalidad de asumir desde la virtualidad, la programación que tenían prevista en lo presencial para el cumplimiento de las metas educativas en el tiempo de pandemia, para este fin además, el (Ministerio de Educación del Ecuador, 2020a), ha gestionado el plan educativo COVID-19, mediante el cual, se proyecta contribuir en la adaptación del docente hacia el uso de la virtualidad, promocionando algunos recursos que podrían ser implementados. 
Lo planteado, busca procurar que el docente de la modalidad presencial, asuma con efectividad el desarrollo del aprendizaje desde el enfoque virtual; siendo pertinente para ello contextualizar los diversos recursos virtuales a las necesidades de cada materia. En el caso de los proyectos de ciencias naturales, se debe planificar con el propósito de que el estudiante trabaje en casa con los recursos cotidianos y que se pueda generar aprendizaje significativo. Para tal fin, el docente debe innovar educativamente y crear las condiciones adecuadas de realización de los proyectos con apoyo de lo virtual; teniendo en cuenta referencias como la presentada por el Ministerio de Educación del Ecuador (2019), en relación a una guía didáctica para realizar proyectos en plataforma virtual.

Lo anterior, sumado al plan educativo COVID-19 del Ministerio de Educación del Ecuador, el docente tiene la oportunidad de diseñar e implementar recursos didácticos en consonancia al desarrollo de proyectos de ciencias naturales por parte de los estudiantes. De este modo, se promueve una educación significativa, articulándose la transcendencia pedagógica del docente (Herrera-Miranda \& Horta-Muñoz, 2016).

El aprendizaje mediado por proyectos, es una estrategia didáctica que permite construir conocimientos en virtud de aprender haciendo e investigando, así (Ramos, Rhea, Pla \& Abreu, 2017), indican que "se necesita crear las condiciones para que puedan ser asimilados todos los componentes del contenido, no basta con informar cómo la sociedad quiere que sean los valores de los educandos" (p. 84). No obstante, este tipo de aprendizaje asumido desde la virtualidad representa un reto para el docente acostumbrado a trabajar en modalidad presencial. Entonces, asumir lo virtual de forma emergente sin contar con experiencia previa en muchos casos, se debe tener en cuenta para diseñar ambientes virtuales de aprendizaje está basado en proyectos; para que estos puedan ser presentado por los estudiantes tanto con pertinencia social como académica, a razón de lo exigido por las autoridades educativas para su debida aprobación.

De igual manera, lo emocional es un aspecto que no puede dejarse de lado y es importante tenerlo en cuenta durante la planificación educativa, incluyéndose actividades en apoyo de la inteligencia emocional de los estudiantes (Salvatierra- 
Tumbaco, Vallejo-Valdivieso \& Moya-Martínez, 2019); por cuanto esto permite favorecer el crecimiento integral de las partes involucradas en el aprendizaje. Por tanto, la mediación tecnológica no puede impedir la generación de interrelaciones sociales basadas en lo asertivo y sinergico como ejes para motivar al logro de las actividades académicas planteadas.

En lo que refiere a la modalidad de aprendizaje basado en proyectos (ABP) es una alternativa que permite al estudiante aprender haciendo desde la realidad social en donde se encuentra inmerso (Travieso-Valdés \&Ortíz-Cardenas, 2018); en este sentido, el docente puede planificar situaciones de aprendizaje en base a la construcción de proyectos de ciencias naturales en razón del contexto del estudiante, brindándole herramientas tanto virtuales como presenciales al alcance en medio de la emergencia sanitaria. En este sentido, el proceso contribuiría a formar la competencia en investigación, así como el pensamiento crítico, propiciándose además, las capacidades metacognitivas de los estudiantes al construir su aprendizaje (MuñozMorales, Barrientos-Oradini, Araya-Castillo \& Reyes-Saavedra, 2019).

Asumir el proceso de esta manera involucra un cambio en el estilo de aprendizaje del estudiante, pero se convierte en una alternativa para que aprenda haciendo (LluchMolins \& Portillo-Vidiella, 2018). Además, tanto docente y estudiantes, promueven el desarrollo de ámbitos que quizás no estarían presentes en la modalidad presencial de estudio, sobre todo cuando esta se basa en lo mecanicista. Uno de estos ámbitos estaría relacionado al aprendizaje critico - autónomo, brindando facultad para conciliar un estudiante reflexivo ante los diversos hechos sociales en su entorno (Collazo-Expósito \&Geli-de-Ciurana, 2017).

El aprendizaje por proyectos, puede incidir positivamente en la motivación de los estudiantes y docentes hacia el logro de la calidad académica (Mora-León, Carranza \& Palíz-Sánchez, 2019). Por tanto, es indispensable tener en cuenta este aspecto para promocionar como recurso didáctico virtual, requiriéndose por parte del docente, conocerlo para poner en práctica, y así propiciar la posibilidad de un abordaje enriquecedor de crecimiento educativo. 
En este contexto, es imprescindible por parte del docente, realizar un proceso de planificación pensando en lo virtual (Pástor, et al., 2018), en el cual además debe tener en consideración los aspectos sociológicos del estudiante, como demografía, acceso a internet, entre otros, (Valverde-Berrocoso \& Balladares-Burgos, 2017); planificación que debe estar ajustada y en consonancia con los recursos de las familias de los estudiantes, asumiéndose que lo económico juega un rol importante el financiamiento de los útiles a emplearse en el proyecto.

Este factor debe ser tenido en cuenta por parte del docente, por cuanto no se trata de generar innovaciones educativas en donde la minoría tenga acceso, al contrario, es necesario que todos los estudiantes puedan acceder a los diversos requerimientos para elaborar el proyecto, en caso contrario, se estaría generando un proceso de exclusión (Cornejo-Espejo, 2019), lo cual no estaría en consonancia con las políticas educativas del Ecuador, donde se proyecta la inclusión como un criterio de calidad educativa.

Otro aspecto a suponer de importancia al momento de planificar, es como generar la socialización entre los estudiantes a pesar de no tener contacto presencial, siendo indispensable por cuanto la educación históricamente ha sido un proceso donde se promueven valores de integración social (Touriñán-López, 2019). Esto permite reflexionar que no solo se trata de enseñar o cumplir determinados contenidos, sino, es necesaria la puesta en práctica de la integración entre pares estudiantiles con la finalidad de promover el trabajo en equipo y cooperativo, a pesar de la educación virtual a distancia.

El aprendizaje mediante el trabajo cooperativo se encuentra ligado al fomento de habilidades socio emocionales en los estudiantes (Estrada, Monferrer \& Moliner, 2016). De este modo, el desarrollo de proyectos en ciencias naturales, representa un desafío durante el período de pandemia, donde los estudiantes deberán construirlo desde la virtualidad, aunado que durante el desarrollo del mismo, se trabaje en relación no solo para aprender temas o contenidos, sino también, el trabajo cooperativo mismo, lo cual permitirá focalizar el desarrollo de habilidades emocionales en medio de una circunstancias nunca antes vivida por la población; y siendo una 
situación educativa que debe contribuir a mejorar la percepción social que puedan tener los estudiantes en relación al afrontamiento social generado en cuarentena por COVID-19.

En este periodo de pandemia, los docentes deben considerar el estrés académico como un factor que podría intervenir durante el proceso de aprendizaje, especialmente concatenado a la ansiedad (Castillo-Pimienta, Chacón-de-la-Cruz \& Díaz-Veliz, 2016), por lo que los proyectos educativos en ciencias naturales, deben ser diseñados pensando en la integración de los estudiantes con sus compañeros y familiares, (Lastre-Meza, López-Salazar \& Alcazar-Berrio, 2018), y para la consecución de un mejor rendimiento académico (Calvo, Verdugo \& Amor, 2016).

Prosiguiendo con lo planteado hasta ahora, en la Unidad Educativa San Francisco Sales, se ha seguido las políticas educativas del Ecuador, asumiéndose la modalidad virtual como escenario para iniciar el año escolar en el régimen Costa - Galápagos 2020 - 2021 (Ministerio de Educación del Ecuador (2020b),por lo que se hace necesario conocer el impacto del uso de los recursos didácticos por parte de los docentes que tienen la responsabilidad de dirigir el proyecto de ciencias naturales, esto con la finalidad de describir en cierto modo, su experiencia pedagógica, por cuanto tradicionalmente han trabajado en la modalidad presencial. A razón de esto, se pretende presentar los resultados obtenidos en la investigación cuyo objetivo estuvo enmarcado en analizar los recursos didácticos virtuales planificados para el abordaje de proyectos educativos de ciencias naturales en período de confinamiento por COVID-19.

\section{MÉTODO}

La investigación se desarrolló en función del paradigma positivista, el cual tiene por esencia observar, medir, describir, aplicándose el método hipotético - deductivo para conocer el objeto que se investiga (Aldana-Zavala, 2019), desde el punto de vista metodológico se apoyó en un trabajo de tipo descriptivo no experimental (Hernández, Fernández \& Baptista, 2014), en conformidad a lo planteado se prosigue el esquema 
de guías STROBE para estudios observacionales para el desarrollo de este apartado (Cartes-Velasquez \& Moraga, 2016).

\section{Población y muestra de estudio}

La población se encuentra conformada por 30 docentes de la Unidad Educativa San Francisco Sales, ubicado en la Provincia de Manabí-Ecuador, en consideración a la muestra, se trabajó con criterios de inclusión, para su debida participación.

\section{Criterios de inclusión de la muestra}

Para ser parte de la muestra de investigación, la población debía cumplir con los siguientes criterios de inclusión:

1. Ser docente del área de ciencias naturales de la Unidad Educativa San Francisco Sales.

2. Estar laborando bajo la modalidad virtual o teletrabajo

3. Estar dispuesto(a) a participar libremente en la investigación, mediante la contestación online de encuesta.

Quienes cumplieron con los criterios mencionados, fueron 6 docentes, constituyéndose en muestra poblacional.

\section{Técnica e instrumento de recolección de información}

Se trabajó con la técnica de la encuesta vía online (formulario Google) con la finalidad que la muestra poblacional aportara información, mediante la aplicación de un cuestionario tipo escala de Lickert constituido por 16 ítems de cinco alternativas de respuestas que van desde 1 a 5 en orden de menor a mayor importancia, lo cual permitió recopilar datos en función del tema de investigación en cumplimiento de las medidas de prevención por COVID-19.

La validación del instrumento se realizó mediante la revisión de contenido por expertos y por el cálculo del alfa de Cronbach, para lo cual fue necesario aplicar una prueba piloto. Para tal fin se aplicó la fórmula propuesta por (Hernández, Fernández \& Baptista, 2014), siendo la siguiente: 


$$
r_{k k}=\frac{k}{k-1}\left[1-\frac{\sum s_{i}^{2}}{s_{t}^{2}}\right]
$$

\section{Dónde:}

$\mathrm{K}$ = número de ítems

$\mathrm{Si}^{2}=$ varianza de los puntajes de cada ítem

$\mathrm{St}^{2}=$ varianza de los puntajes totales

Los datos recopilados fueron calculados en procesador de datos SPSS V25, obteniéndose un resultado de confiabilidad de 0,87 de coeficiente de Cronbach, lo cual se cataloga como confiable para su aplicación.

\section{Análisis de la información recopilada}

Se procedió a aplicarse la estadística descriptiva para el análisis de la información, construyéndose así tablas y gráficos que permitieron analizar los datos y estructurar los resultados en función de cumplir con el objetivo de investigación, para tal fin se apoyó en el programa estadístico SPSS V25. 


\section{RESULTADOS}

\section{Cuadro 1}

Recursos didácticos audiovisuales en redes sociales

\begin{tabular}{ccc} 
Indicador & Fr & $\%$ \\
\hline Video en YouTube & 2 & 10 \\
Video en Facebook & 1 & \\
Video en Instagram & 2 & 20 \\
Video por wasap & 1 & 30 \\
Total & 6 & 100
\end{tabular}

Fuente: A partir de recolección de información.

El 10\% de la población destaca implementar el Video en YouTube, el $20 \%$ usa el Video en Facebook, el 30\% emplea el Video en Instagram y el $40 \%$ implementa el Video por wasap como recurso didáctico para promover el aprendizaje.

\section{Cuadro 2}

Aulas virtuales

\begin{tabular}{rrrr}
\multicolumn{2}{c}{ Indicador } & $\mathrm{Fr}$ & $\%$ \\
\hline Google classroom & 1 & 19 \\
Edmodo & 2 & 23 \\
Zoom & 1 & 27 \\
Total & Moodle & 2 & 31 \\
\hline
\end{tabular}

Fuente: A partir de recolección de información.

El 19\% emplea el Google classroom, el 23\% Edmodo, el 27\% indica que implementa Zoom y el $31 \%$ indica que usa Moodle como plataformas educativas para el diseño de ambientes virtuales de aprendizaje. 


\section{Cuadro 3}

Método de aprendizaje

\begin{tabular}{llr}
\multicolumn{1}{c}{ Indicador } & Fr & $\%$ \\
\hline Clase invertida & 2 & 21 \\
Aprendizaje basado en casos & 1 & 24 \\
Aprendizaje basado en problemas & 1 & 26 \\
Aprendizaje basado en proyectos & 2 & 29 \\
Total & 6 & 100 \\
\hline
\end{tabular}

Fuente: A partir de recolección de información.

\section{Cuadro 4}

Plataformas de ciencias naturales

\begin{tabular}{llr}
\multicolumn{1}{c}{ Indicador } & Fr & $\%$ \\
\hline National Geographic "Aprende en casa" & 3 & 22 \\
Plataforma educativa Santillana & 1 & 24 \\
Discovery en la escuela & 1 & 26 \\
Diseñada por el docente & 1 & 28 \\
Total & 6 & 100 \\
\hline
\end{tabular}

Fuente: A partir de recolección de información.

El 22\% se apoya en National Geographic "Aprende en casa”, el 24\% lo hace mediante la Plataforma educativa Santillana, el $26 \%$ indica que proyecta usar Discovery en la escuela y el $28 \%$ prefiere diseñar su propia plataforma educativa con fines de enseñar sobre ciencias naturales. 


\section{DISCUSIÓN}

En cuanto a los recursos didácticos audiovisuales en redes sociales (Barros-Bastida \& Barros-Morales, 2015), indican que "los medios audiovisuales tienen una importancia trascendental desde el punto de vista didáctico, debe asumirse por cada académico y se debe enseñar a partir de los mismos" (p. 31), por consiguiente implementar su uso adecuado como elemento para propiciar un aprendizaje significativo en las ciencias naturales, siendo el video fundamental para tal fin (GarcíaMatamoros, 2014), por cuanto permite que los estudiantes recopilen y analicen información en función de material documental que promueve procesos mentales en concordancia de promover un aprendizaje duradero.

Esto, aunado a que además el estudiante tiene la oportunidad de grabar videos en donde debe demostrar la comprensión de un determinado tema, con la finalidad de suministrar información pertinente, el cual además debe ser estructurado para una transmisión efectiva del mensaje, lo cual concuerda con (Rodríguez-Licea, LópezFrías \& Mortera-Gutiérrez, 2017), quienes destacan en su investigación que los estudiantes logran una mejor aprehensión conceptual y procedimental de los temas abordados en clase.

El segmento sobre aulas virtuales es de suma importancia por cuanto permite diseñar el micro currículo con la finalidad de establecer un proceso de aprendizaje significativo (Islas-Torres, 2017), teniendo en cuenta que el mundo ha cambiado y es urgente la implementación de lo virtual como escenario para establecer el sistema educativo en razón de la cotidianidad acontecida por el COVID-19, siendo necesario el acompañamiento de las familias en el aprendizaje de los estudiantes con la finalidad de contar con apoyo moral para propiciar motivaciones al logro (Páez, 2018).

Así mismo, es conveniente alertar sobre la necesidad de capacitación sobre el uso de aulas virtuales tanto para docentes como estudiantes, e incluso familiares que acompañan en el proceso de aprendizaje, por cuanto (Monroy, Hernández \& Jiménez, 2018), indican que podría existir predisposición al empleo de las mismas, lo cual podría repercutir negativamente en el rendimiento académico, más aun, cuando los actores involucrados provienen de la modalidad presencial, no estando habituados a 
lo virtual con fines académico, existiendo un proceso de transición que debe ser promovido con base a la formación continua para evitar deserciones y fracasos escolares.

El método de aprendizaje a implementar durante el periodo académico en virtualidad, permite que el estudiante tome mayor responsabilidad en su aprendizaje, mediante la investigación e indagación que debe realizar (Silva-Paim, Thauana-lappe \& Brandalize-Rocha, 2014). Así mismo, este enfoque se centra en el estudiante, situación que contribuye a tener mayor participación que en el modelo tradicional (Hernández-Silva \& Tecpan-Flores, 2018).

El enfoque centrado en el estudiante, permite promover mayor responsabilidad al discente con la finalidad de que el docente se constituya en un asesor del proceso (Burgos-Briones, Alvarado-Pino \& Valdez-Guerrero, 2019), promoviéndose la búsqueda, indagación investigativa como estrategia fundamental para la construcción del aprendizaje, siendo así concordante con el paradigma constructivista social del conocimiento.

La enseñanza de las ciencias naturales, debe generarse en interconectividad de plataformas que contribuyan el fomento de un aprendizaje integrador, promoviéndose la vinculación de lo teórico con la cotidianidad que rodea al estudiante, de esa forma, el saber se constituye como un eje fundamental para una adecuada conformación de una persona reflexiva (Jaramillo-Naranjo, 2019), sin embargo, enseñar bajo esta modalidad lo referente al proyecto de ciencias naturales, es un reto por cuanto se experimenta un proceso que genera incertidumbre pero además un aprendizaje que determinará nuevos procesos a seguir para lograr un mejor aprendizaje (Busquets, Silva \& Larrosa, 2016).

\section{CONCLUSION}

Los recursos didácticos virtuales proyectados por los docentes para ser implementados en el proyecto educativo de ciencias naturales, son idoneos para promover un aprendizaje significativo en los estudantes, sin embargo, es necesario contar con un proceso de capacitación tanto para profesores como discentes con la 
finalidad de evitar inconvenientes que perjudiquen el aprendizaje, así como el rendimiento escolar.

Así mismo, la investigación presenta avances importantes, requiriendose proseguir una segunda fase en donde se pueda determinar el impacto sobre los estudiantes y docentes, por el uso de la implementación virtual de aprendizajes en sustitución de lo presencial por motivos de la pandemia COVID- 19, lo cual permitirá contar con resultados generalizables y comparativos con investigaciones similares a la planteada, teniendose hasta ahora como limitación que el nuevo período escolar recien inicia, por lo que como se ha comentado es menester medir a mediados o final del período la efectividad de los recursos didácticos implementados.

\section{FINANCIAMIENTO}

No monetario

\section{AGRADECIMIENTOS}

Al personal Docente de la Unidad Educativa San Francisco Sales, ubicado en la Provincia de Manabí-Ecuador; por el apoyo en la realización de la investigación.

\section{REFERENCIAS CONSULTADAS}

Abreu, O, Gallegos, M, Jácome, J, \& Martínez, R. (2017). La Didáctica: Epistemología y Definición en la Facultad de Ciencias Administrativas y Económicas de la Universidad Técnica del Norte del Ecuador. [Didactics: Epistemology and Definition in the Faculty of Management and Economics at the Technical University of Northern Ecuador]. Formación universitaria, 10(3), 8192. https://dx.doi.org/10.4067/S0718-50062017000300009

Aldana-Zavala, J. J. (2019). La competencia epistemológica en el investigador social universitario venezolano. [Epistemological competence in the Venezuelan university social researcher]. Praxis, 15(1), 103-115. https://doi.org/10.21676/23897856.3091 
Barros-Bastida, M. C., \& Barros-Morales, D. C. R. (2015). Los medios audiovisuales y su influencia en la educación desde alternativas de análisis. [Audiovisual aids and their influence in education from alternatives of analysis]. Universidad y Sociedad, 7(3), 26-31. Recuperado de https://url2.cl/jMgN5

Burgos-Briones, J., Alvarado-Pino, L., \& Valdez-Guerrero, R. (2019). Enfoque escolar centrado en el estudiante. [Student-centered school approach]. CIENCIAMATRIA, 5(1), 45-58. https://doi.org/10.35381/cm.v5i1.240

Busquets, T, Silva, M, \& Larrosa, P. (2016). Reflexiones sobre el aprendizaje de las ciencias naturales: Nuevas aproximaciones y desafíos. [Reflections on the Teaching-Learning of the Natural Sciences: New perspectives and challenges]. Estudios pedagógicos (Valdivia), 42(especial), 117135. https://dx.doi.org/10.4067/S0718-07052016000300010

Calvo, Mํㅗ I, Verdugo, M, \& Amor, A. (2016). La Participación Familiar es un Requisito Imprescindible para una Escuela Inclusiva. [Family Participation is an Essential Requirement for an Inclusive School]. Revista latinoamericana de educación inclusiva, $\quad 10(1), \quad 99-113 . \quad$ https://dx.doi.org/10.4067/S0718$\underline{73782016000100006}$

Castillo-Pimienta, C, Chacón-de-la-Cruz, T, \& Díaz-Veliz, G. (2016). Ansiedad y fuentes de estrés académico en estudiantes de carreras de la salud. [Anxiety and sources of academic stress in students of health careers]. Investigación en educación médica, 5(20), 230237. https://dx.doi.org/10.1016/j.riem.2016.03.001

Cartes-Velasquez, R, \& Moraga, J. (2016). Pautas de chequeo, parte III: STROBE y ARRIVE. [Cheklists, part III: STROBE and ARRIVE]. Revista chilena de cirugía, 68(5), 394-399. https://dx.doi.org/10.1016/j.rchic.2015.12.003

Collazo-Expósito, L. \&. Geli-de-Ciurana, A. (2017). Avanzar en la educación para la sostenibilidad. Combinación de metodologías para trabajar el pensamiento crítico y autónomo, la reflexión y la capacidad de transformación del sistema. [Advance in education for sustainability. Combination of methodologies to work critical and autonomous thinking, reflection and the capacity for transformation of the system]. Revista Iberoamericana De Educación, 73, 131-154. https://doi.org/10.35362/rie730295

Cornejo-Espejo, J. (2019). Nuevos excluidos en el sistema educacional chileno: problemas y desafíos. [New Excluded in the Chilean Educational System: Problems and Challenges]. Páginas De Educación, 12(1), 28-48. https://doi.org/10.22235/pe.v12i1.1766 
Estrada, M, Monferrer, D, \& Moliner, M. (2016). El Aprendizaje Cooperativo y las Habilidades Socio-Emocionales: Una Experiencia Docente en la Asignatura Técnicas de Ventas. [Cooperative Learning and Socio-Emotional Skills: A Teaching Experience in the Sales Techniques Subject]. Formación universitaria, 9(6), 43-62. $\quad$ https://dx.doi.org/10.4067/S0718$\underline{50062016000600005}$

García-Matamoros, M. A. (2014). Uso Instruccional del video didáctico. Revista de Investigación, 38 (81), 43-67. [Instructional use of the teaching video]. Recuperado de https://url2.cl/Dg8Qv

Hernández, R., Fernández, C., \& Baptista, M. (2014). Metodología de la Investigación. [Research Methodology]. Mexico DF: McGraw-Hill / Interamericana Editores, S.A. DE C.V.

Hernández-Silva, C., \& Tecpan-Flores, S. (2018). Aula invertida mediada por el uso de plataformas virtuales: un estudio de caso en la formación de profesores de física. [Inverted classroom mediated by the use of virtual platforms: a case study in the training of physics teachers]. Estudios Pedagógicos, 43(3), 193-204. https://doi.org/10.4067/S0718-07052017000300011

Hernández, A. (2018). Transitando por el camino de la escuela para padres, madres $y$ representantes. Una experiencia vivida. [Walking on the way to school for parents and representatives. A lived experience]. EPISTEME KOINONIA, 1(1), 51-71. http://dx.doi.org/10.35381/e.k.v1i1.490

Herrera-Miranda, G., \& Horta-Muñoz, D. (2015). La superación pedagógica y didáctica, necesidad impostergable para los profesores y tutores del proceso de especialización. [The pedagogical and educational self-improvement, an imperative need for teachers and advisors under the specialization process]. Educación Médica Superior, 30(3). Recuperado de https://url2.cl//bhzD2

Islas-Torres, C. (2018). La implicación de las TIC en la educación: Alcances, Limitaciones y Prospectiva [The role of ICT in education: Applications, Limitations, and Future Trends]. RIDE Revista Iberoamericana Para La Investigación y El Desarrollo Educativo, 8(15), 861 - 876. https://doi.org/10.23913/ride.v8i15.324

Jaramillo-Naranjo, L. (2019). Las ciencias naturales como un saber integrador. [Natural Sciences as an integrating knowledge]. Sophia, Colección de Filosofía de la Educación, (26), 199221. https://dx.doi.org/10.17163/soph.n26.2019.06 
Lastre-Meza, K., López-Salazar, L. D., \& Alcazar-Berrio, C. (2018). Relación entre apoyo familiar y el rendimiento académico en estudiantes colombianos de educación primaria. [Relationship between family support and academic achievement in primary school students]. Psicogente, 21(39), 102-115. https://doi.org/10.17081/psico.21.39.2825

Lattá-Arias, C. (2019). Uso de las TIC para proyectos productivos en las instituciones educativas del Municipio Zona Bananera. Magdalena. Colombia. [Use of ICT for productive projects in educational institutions of the Municipality of the Banana Zone. Cupcake Colombia]. Revista Arbitrada Interdisciplinaria Koinonía, 4(7), 233-246. http://dx.doi.org/10.35381/r.k.v4i7.202

Lluch-Molins, L., \& Portillo-Vidiella, M. C. (2018). La competencia de aprender a aprender en el marco de la educación superior. [The competence of learning to learn in the framework of higher education]. Revista lberoamericana De Educación, 78(2), 59-76. https://doi.org/10.35362/rie782318

Ministerio de Educación del Ecuador (2020a). Plan educativo COVID-19. [COVID-19 educational plan]. Recuperado de https://url2.cl/vGwNr

Ministerio de Educación del Ecuador (2020b). Las clases en el régimen CostaGalápagos 2020-2021, iniciarán el 01 de junio de 2020 de manera no presencial. [Classes in the Costa-Galápagos regime 2020-2021, will begin on June 1, 2020, in person]. Recuperado de https://url2.cl/qfunX

Ministerio de Educación de la Nación (2019). Laboratorio virtual de ciencias. [Virtual science laboratory]. Recuperado de https://url2.cl/6gTJe

Ministerio del Trabajo (2020). Acuerdo Ministerial Nro. MDT-2020-076. [Ministerial Agreement No. MDT-2020-076]. Recuperado de https://url2.cl//kbh8

Mora-León, W., Carranza, L., \& Palíz-Sánchez, C. (2019). El aprendizaje basado en proyecto: Realidad y perspectivas. [Project-based learning: Reality and perspectives]. Journal of Science and Research: Revista Ciencia e Investigación. 4(4), 22 - 33. Recuperado de https://url2.cl/RAWEr

Monroy, A, Hernández, I, \& Jiménez, M. (2018). Aulas digitales en educación superior: el caso de México. [Digital Classrooms in Higher Education: The Case of Mexico]. Formación universitaria. 11 (5), $93-$ 104. https://dx.doi.org/10.4067/S0718-50062018000500093

Muñoz-Morales, N., Barrientos-Oradini, N., Araya-Castillo, L., \& Reyes-Saavedra, J. (2019). Capacidades metacognitivas en el sistema educativo en instituciones educativas de educación media. Revista Arbitrada Interdisciplinaria Koinonía, 4(7), 103-127. http://dx.doi.org/10.35381/r.k.v4i7.196 
Páez, A. (2018). Docentes y padres en el proceso de aprendizaje de los estudiantes. [Teachers and parents in the student learning process]. EPISTEME KOINONIA, 1(2), 18-34. http://dx.doi.org/10.35381/e.k.v1i2.509

Pando, V. (2018). Tendencias didácticas de la educación virtual: Un enfoque interpretativo. [Teaching trends in virtual education: An interpretive approach]. $\begin{array}{llll}\text { Propósitos } y & \text { Representaciones, }\end{array}$ http://dx.doi.org/10.20511/pyr2018.v6n1.167

Pástor, D, Jiménez, J, Arcos, G, Romero, M, \& Urquizo, L. (2018). Patrones de diseño para la construcción de cursos on-line en un entorno virtual de aprendizaje. [Design patterns for building online courses in a virtual learning environment]. Ingeniare. Revista chilena de ingeniería, 26(1), 157171. https://dx.doi.org/10.4067/S0718-33052018000100157

Rama, C. (2016). La fase actual de expansión de la educación en línea o virtual en América Latina. [The current phase of expansion of online or virtual education in Latin America].Universidades, (70), 27-39. Recuperado de https://url2.cl/YSxMQ

Ramos, J, Rhea, B, Pla, R, \& Abreu, O. (2017). La Pedagogía como Ciencia para el Tratamiento de los Contenidos Generales del Proceso Educativo y la Formación de Valores. [Pedagogy as a Science for the Treatment of the General Contents of the Educational Process and the Formation of Values]. Formación universitaria, 10(6), 77-86. https://dx.doi.org/10.4067/S0718$\underline{50062017000600009}$

Rodríguez-Licea, R, López-Frías, B, \& Mortera-Gutiérrez, F. (2017). El video como Recurso Educativo Abierto y la enseñanza de Matemáticas. [Videos as an Open Educational Resources and Mathematics Teaching]. Revista electrónica de investigación educativa, 19(3),

92100. https://dx.doi.org/10.24320/redie.2017.19.3.936

Salvatierra-Tumbaco, D., Vallejo-Valdivieso, P., \& Moya-Martínez, M. (2019). Estructuras mentales y competencias emocionales en estudiantes universitarios. [Mental structures and emotional competencies in university students]. CIENCIAMATRIA, 5(1), 118-132. https://doi.org/10.35381/cm.v5i1.260

Silva-Paim, A. Thauana-lappe, N, \& Brandalize-Rocha, D. (2014). Métodos de enseñanza utilizados por docentes del curso de enfermería: enfoque en la metodología de investigación. [Teaching methods used by teachers of the nursing course: focus on research methodology]. Enfermería Global, 14(1), 136-169. https://doi.org/10.6018/eglobal.14.1.186291 
Maira Dolores Vélez-Loor; Patricio Alfredo Vallejo-Valdivieso; María Elena Moya-Martínez

Torres, C, \& Rodríguez, J. (2019) Los entornos de aprendizaje inmersivo y la enseñanza a ciber-generaciones. [Immersive learning environments for teaching the cyber generations]. Educação e Pesquisa, 45, e187369. Epub 19 de junio de 2019. https://doi.org/10.1590/s1678-4634201945187369

Touriñán-López, J. (2019). La relación educativa es un concepto con significado propio que requiere concordancia entre valores y sentimientos en cada interacción. [The educational relationship is a concept with own meaning, that it requires concordance between values and feelings in each interaction]. Sophia, Colección de Filosofía de la Educación, (26), 223279. https://dx.doi.org/10.17163/soph.n26.2019.07

Travieso-Valdés, D. \& Ortíz-Cardenas, T. (2018). Aprendizaje basado en problemas y enseñanza por proyectos: Alternativas diferentes para enseñar. Revista Cubana de Educación Superior, 37(1), 124-133.

Valverde-Berrocoso, J, \& Balladares-Burgos, J. (2017). Enfoque sociológico del uso del b-learning en la educación digital del docente universitario. [Sociological approach to the use of b-learning in digital education of university teachers]. Sophia, Colección de Filosofía de la Educación, (23), 123140. https://dx.doi.org/10.17163/soph.n23.2017.04

Vallejo Valdivieso, P., Zambrano Pincay, G., Vallejo Pilligua, P., \& Bravo Cedeño, G. (2019). Modelos de planificación educativa y diversidad en aulas de clases. [Models of educational planning and diversity in classrooms]. CIENCIAMATRIA, 5(9), 302-315. https://doi.org/10.35381/cm.v6i10.149 